\title{
The Role of Customer Readiness in User's Willingness to Use Augmented Reality: An Abstract
}

\author{
Atieh Poushneh
}

\begin{abstract}
As the number of augmented reality applications increases, it will be necessary to acknowledge how customers become prepared to use this technology. Since prior research barely emphasized the role of customer readiness in customers' willingness to use augmented reality, this study develops a conceptual framework regarding how customers become ready to use augmented reality. Drawing on the intrinsic motivation theory and the diffusion of innovation theory, this study presents that customer readiness is a recipient of various antecedents, including personal innovativeness, previous experience, desire for control, need for interaction, need for touch, personal innovativeness, service complexity, perceived risk, relative advantage, perceived playfulness, perceived interactivity, and novelty, and influences customers' willingness to use augmented reality.
\end{abstract}

\footnotetext{
A. Poushneh $(\bowtie)$

University of Texas Rio Grande Valley, Rio Grande City, TX, USA

e-mail: atieh.poushneh01@utrgv.edu
} 